\title{
ORIENTAÇÕES PARA SUBMISSÃO E PUBLICAÇÃO NA REVES: O TEMPLATE
}

\author{
SUBMISSION GUIDELINES: REVES TEMPLATE
}

Roberta Ferreira Coelho de Andrade ${ }^{1}$, Wagner dos Reis Marques Araújo², Aldair Oliveira de Andrade $^{3}$ e Antonio Marcos de Oliveira Siqueira ${ }^{4}$

1 Universidade Federal do Amazonas, Departamento de Serviço Social, Manaus/AM, Brasil, E-mail: roberta ufam@yahoo.com.br, ORCID: https://orcid.org/0000-00026535-0760

${ }^{2}$ Universidade do Estado de Minas Gerais, UEMG - Unidade Abaeté, MG Brasil, Email: wagner.araujo@uemg.br, ORCID: https://orcid.org/0000-0003-0793-0043

3 Universidade Federal do Amazonas, Programa de Pós-Graduação em Ciências e Humanidades (PPGCH), Brasil, E-mail: aldairandrade@yahoo.com.br, ORCID: https://orcid.org/0000-0001-5205-9766

4 Universidade Federal de Viçosa, Viçosa, MG, Brasil, E-mail: antonio.siqueira@ufv.br, ORCID: https://orcid.org/0000-0001-9334-0394

A RTICLE INFO

Article history:

Received 2019-12-14

Accepted 2019-12-14

Available online 2019-12-14
Palavras-chave: Normas de publicação. Template. Periódico. Manuscrito.

Keywords: Guide for Authors. Template. Journal. Manuscript paper.

RESUMO. O Resumo deve ser apresentado na língua do artigo, de 150 a 250 palavras e deve ser escrito num único parágrafo. A fonte é ARIAL 12, espaçamento simples, sem recuo, com espaço de 0 pontos antes, 0 pontos depois, justificado. $O$ resumo deve apresentar o problema, o(s) objetivo(s), o método, os resultados e a conclusão/contribuição do trabalho. $O$ artigo já deve ser submetido para avaliação conforme este template de artigo final. Os autores aceitam que a REVES efetue, nos originais aprovados para publicação, revisão e alterações de ordem normativa, ortográfica e gramatical. Os autores concedem a REVES o direito de primeira publicação da versão revisada do artigo. A REVES avalia: (i) a organização do texto e sua redação; (ii) a qualidade técnica e científica do trabalho e (iii) a relevância para a área em questão. Quanto a organização do texto observa-se a estruturação das seções, a declaração dos objetivos, a transição entre seções e a relação coerente entre a introdução e conclusão. Observa-se também o atendimento a normas de composição do resumo, citação e referência de fontes seguindo as Normas da ABNT. Com relação a qualidade técnica e científica do trabalho, avalia-se o suporte consistente da fundamentação empregada relacionando 0 trabalho a fontes bibliográficas atuais e referências. Avalia-se também a coerência do método adotado para o delineamento e desenvolvimento do trabalho. Quanto à identificação da relevância do trabalho ao tema relacionado avalia-se a originalidade e contribuição do mesmo para ampliar o conhecimento na área. Deve-se evitar, no resumo: abreviaturas, símbolos, e demais elementos que não sejam absolutamente 
necessários à sua compreensão, bem como comentários, críticas e julgamento pessoal; palavras e/ou expressões supérfluas.

ABSTRACT. An abstract in English always is included when the article is in Portuguese or Spanish. If the article is in English than first the abstract is presented followed by the Resumo in Portuguese. The abstract is 150-250 words long and should be written in a single paragraph. The letter source is ARIAL 12, single-spaced, without indentation, with 0 points of space before, 0 after, justified. The abstract should present the problem, purpose, method, results and scientific contribution of the research. The article must be submitted in the final publishing template. The authors accept that the REVES apply on the article approved for publication, revision and amendment of standards, spelling and grammar. The authors grant REVES the right of the first publication of the revised version of the article. The REVES evaluates: (i) the organization of the text and its writing; (ii) the technical and scientific quality of the work and (iii) the relevance to the topic. The organization of the text and writing considers the article structure, objectives declaration, transition between sections and consistent relationship between the introduction and conclusion. It is also observed the care of composition of abstract, citation and referencing rules of literature sources following the ABNT standard. Regarding the technical and scientific quality of the work, reviewers evaluate the consistent theoretical foundations employed relating to current literature and the coherence of the method adopted. The relevance of the work is related to its originality and contribution to the knowledge in the area.

\section{Introdução}

(Recomenda-se o conhecimento prévio dos TUTORAIS, disponíveis no link https://periodicos.ufv.br/reves/Tutorials, bem como de INFORMAÇÕES ADICIONAIS - Equipe Editorial, Qualis, Taxas de Publicação, etc. - a respeito do periódico, disponíveis no link https://periodicos.ufv.br/reves/about).

(Após a submissão, em caso de necessidade, o contato com a Editoria do periódico deverá ser estabelecido por meio do endereço eletrônico reves.journal@ufv.br, informando no campo ASSUNTO da mensagem o número da submissão - uma identificação única para cada artigo, representada por um número que está disponível no sistema OJS, e informado em mensagens automáticas do sistema).

(Seguir as orientações

disponíveis

no

link: https://periodicos.ufv.br/reves/about/submissions em Diretrizes para Autores).

A intenção deste template é padronizar a formatação dos artigos encaminhados à REVES - Revista Relações Sociais, com vistas a melhorar a qualidade dos trabalhos, buscando a excelência na publicação científica. Assim, tais padrões estão adequados às revistas e aos critérios de indexação da base SciELO (Scientific Electronic Library Online). Este plano de ação tem como objetivos principais: a) Facilitar a correção dos textos; b) Auxiliar o autor no processo de formatação; c) Adequação da periodicidade das publicações aos critérios SciELO; d) Fortalecer o Comitê Editorial da REVES - Revista Relações Sociais; e) Padronizar o estilo e layout da revista (diagramação e conteúdo, capa e sumário).

Para atender o objetivo de padronização de estilo e layout, apresenta-se neste documento o modelo de formatação de artigo científico criado para o programa 
Microsoft Word. Portanto, os artigos prontos devem ser submetidos utilizando o padrão descrito neste documento.

As normas de publicação da REVES, disponíveis no site <https://periodicos.ufv.br/ojs/reves>, estão embutidas nos formatos apresentados neste modelo. Vale lembrar que os artigos serão submetidos à avaliação dos membros da Comissão Científica da revista e de pareceristas ad hoc. Se necessário, os artigos serão encaminhados novamente aos autores para revisão. A versão final do artigo será disponibilizada no site da revista no formato Portable Document Format - PDF. A adoção de um modelo para os artigos possui benefícios que vão além da simplificação do trabalho dos autores. Uma vez que a equipe editorial da revista receba os artigos prontos, ou com baixos índices de inconformidades, reduzse consideravelmente o prazo de editoração, permitindo adequar a periodicidade das edições aos padrões da base SciELO.

\section{Desenvolvimento}

A REVES - Revista Relações Sociais é uma publicação semestral, cujo objetivo é publicar trabalhos inéditos sobre temas relevantes das Ciências Sociais, mantendo uma linha interdisciplinar, com contribuições da Sociologia, da Antropologia e da Ciência Política, bem como de áreas afins.

(Recomenda-se o conhecimento prévio dos TUTORAIS, disponíveis no link https://periodicos.ufv.br/reves/Tutorials, bem como de INFORMAÇÕES ADICIONAIS - Equipe Editorial, Qualis, Taxas de Publicação, etc. - a respeito do periódico, disponíveis no link https://periodicos.ufv.br/reves/about).

OBS.: Trabalhos apresentados anteriormente em eventos científicos da área, cujo resumo e/ou versão integral tenham sido publicados em anais, podem ser submetidos ao periódico, cabendo ao Corpo Editorial a decisão de publicá-los, desde que o autor informe durante o processo de submissão, o nome do evento, data e local em que foram apresentados e anexe o Certificado de Apresentação/Publicação do trabalho no evento (como Documento Suplementar). Recomenda-se que a versão a ser publicada no periódico agregue as discussões e avanços obtidos com a discussão do trabalho no evento científico em que foi apresentado anteriormente. A publicação de artigos está condicionada a pareceres de membros do Conselho Científico e/ou de colaboradores ad hoc. Todos os textos enviados para a revista serão submetidos a um processo de avaliação, realizada por pares, ficando preservada pelo Conselho Editorial a identificação dos avaliadores e dos autores.

A publicação de artigos está condicionada a pareceres de membros do Conselho Científico e/ou de colaboradores ad hoc. Todos os textos enviados para a revista serão submetidos a um processo de avaliação, realizada por pares, ficando preservada pelo Conselho Editorial a identificação dos avaliadores e dos autores.

$\mathrm{O}$ artigo deve ser formatado através do programa Word for Windows, com a configuração da página com tamanho de papel A4, com as margens iguais a 2,0, 2,5, 2,0 e e 2,5 cm (como neste texto), em coluna única. Os artigos completos devem ser enviados em dois formatos: .PDF e WORD (.docx). Sugerimos texto com até no máximo 15 páginas, conforme especificação do formato, considerando as apresentações, imagens, gráficos, tabelas, notas e referências. As figuras devem ser inseridas no texto com quebra de texto "inferior e superior" e posição "alinhada com o texto". 
Verifique os exemplos no corpo deste modelo. Todas as imagens devem ser em 300 dpi (ou superior). As imagens precisam ser "chamadas" no texto (Figura 1). A introdução representa o contato inicial do leitor com o texto, portanto, deve ser clara, objetiva e sintética. Nela se trata sucintamente da natureza do assunto, dos objetivos, das limitações, dos métodos e resultados do trabalho apresentado. A primeira página do artigo deve incluir: títulos, nomes dos autores, instituição de afiliação do autor com cidade, estado, país, E-mail do autor de contato, Resumo, Palavras-chave, Abstract, Keywords, conforme este template. O resumo e abstract devem conter de 150-250 palavras cada um. Entretanto, deve-se ajustar o tamanho dos mesmos, de tal forma que os elementos sejam apresentados somente na $1^{\text {a }}$ página e tenham a aparência final, como esta do template. A primeira página não deverá conter nenhuma informação além daquelas definidas. O corpo do artigo deverá apresentado após o traço destacado em preto.

\section{Formatação Geral}

(FAVOR NÃO EDITAR NOS CAMPOS DESTACADOS EM VERMELHO. MANTER COMO ESTÃO). A formatação do artigo deve fazer uso exclusivamente dos estilos conforme apresentado neste template. O corpo do texto deve ser escrito em fonte é ARIAL 12, espaçamento SIMPLES, 1,0, sem recuo, com espaço de 0 pontos antes, 0 pontos depois, justificado, com Tabulação ( $1^{\text {a }}$ linha, com 1,27 cm). A $1^{\text {a }}$ página, antes do elemento textual Introdução segue uma formatação diferenciada, conforme este template. Pode-se utilizar até três níveis de seções. Os títulos das seções devem ser em negrito, alinhados a esquerda, conforme apresentado neste template. Não deixe espaços vazios ao longo do texto. Distribua o texto de modo a se ter apresentação harmônica, como a deste template. $O$ cabeçalho apresentado na $1^{a}$ página, antes do título deve figurar exatamente como consta no presente documento (e não deve ser editado pelos autores), bem como a paginação, que não deve ser alterada, como neste modelo. O campo ARTICLE INFO não deve ser preenchido pelos autores. Preencha apenas a data de submissão do artigo, na parte destacada em amarelo.

\subsection{Estruturação do texto}

Sempre que possível, orienta-se estruturar o texto nas seções: (i) Introdução,

(ii) Fundamentação ou Revisão, (iii) Método/Metodologia/Procedimentos Metodológicos, (iv) Resultados e Discussão, (v) Conclusão ou Considerações Finais, (vi) Agradecimentos, (vii) Referências.

$\mathrm{Na}$ introdução deve-se contextualizar a pesquisa e declarar os objetivos do estudo apresentado. Trata-se de uma seção obrigatória.

$\mathrm{Na}$ Fundamentação deve-se apresentar a teoria ou trabalhos correlatos que dão embasamento ao enfoque da questão abordada e à discussão da mesma.

$\mathrm{Na}$ seção de Método deve-se declarar o tipo de método adotado, seu delineamento, abrangência e limites da pesquisa. Também na seção de Método deve-se esclarecer como os dados serão analisados.

$\mathrm{Na}$ seção de resultados e discussão deve-se apresentar o desenvolvimento do estudo, os dados coletados e discuti-los segundo o método apresentado visando atingir o objetivo declarado.

$\mathrm{Na}$ Conclusão/ Considerações Finais deve-se fazer uma síntese dos resultados e apontar a contribuição atingida. Geralmente o tipo da pesquisa ou 
trabalho tem implicações sobre a estrutura do texto, portanto, cabe aos autores a estruturação do texto em seções pertinentes. A seção Agradecimentos não é obrigatória. A seção final, Referências, é obrigatória e nela são apresentadas todas as referências utilizadas no manuscrito. Não escrever Referências Bibliográficas. Escreva apenas REFERÊNCIAS.

\subsection{Idiomas}

Podem ser submetidos artigos e resenhas nos idiomas português, inglês, francês e espanhol, que, se aprovados, serão publicados na língua original. Os custos com revisão de artigos em inglês, francês e espanhol deverão ser cobertos pelo(a) autor(a). A REVES indicará os profissionais habilitados. As submissões poderão receber pareceres nas três línguas. Ao submeter o artigo, o(a) autor(a) concorda em receber pareceres nas três línguas.

\section{Processo de Submissão}

(Recomenda-se o conhecimento prévio dos TUTORAIS, disponíveis no link https://periodicos.ufv.br/reves/Tutorials, bem como de INFORMAÇÕES ADICIONAIS - Equipe Editorial, Qualis, Taxas de Publicação, etc. - a respeito do periódico, disponíveis no link https://periodicos.ufv.br/reves/about).

Para publicação do artigo na REVES seguem-se as seguintes etapas: a) Cadastramento de um dos autores no sistema da revista, b) Submissão online do artigo, c) Avaliação geral dos editores; d) Processo de Avaliação às Cegas por Pares; e) Comunicação com/aos autores; f) Edição do texto para publicação (caso aceito); g) Publicação do artigo no sítio do periódico.

\subsection{Cadastro de um dos autores}

Um dos autores do artigo deve se cadastrar como autor no sistema da revista (https://periodicos.ufv.br/ojs/reves/user/register). Se já se cadastrou como Autor, deverá iniciar o processo de submissão, por meio do login no sistema: https://periodicos.ufv.br/ojs/reves/login. Este autor será responsável pela submissão do artigo online e somente este autor terá acesso ao sistema para acompanhar o processo de avaliação e edição do artigo. Recomenda-se que todas informações solicitadas no cadastro sejam fornecidas. Orienta-se que no campo URL seja inserido o link para o CV Lattes, ResearchID ou ORCiD (Open Researcher and Contributor ID). Recomenda-se também incluir telefone para contato. O Autor da Submissão (aquele que irá submeter o manuscrito, independentemente de ser o autor principal) deve informar no cadastramento todas as informações marcadas com asterisco.

\subsection{Submissão online}

(Seguir as orientações disponíveis no link: https://periodicos.ufv.br/reves/about/submissions em Diretrizes para Autores).

\section{Processo de Avaliação}

(Recomenda-se o conhecimento prévio dos TUTORAIS, disponíveis no link https://periodicos.ufv.br/reves/Tutorials, bem como de INFORMAÇÕES ADICIONAIS 
- Equipe Editorial, Qualis, Taxas de Publicação, etc. - a respeito do periódico, disponíveis no link https://periodicos.ufv.br/reves/about).

\subsection{Avaliação às Cegas pelos Pares}

Os trabalhos submetidos serão encaminhados para apreciação de dois a três pareceristas (ad hoc), especialistas nas áreas de atuação do periódico, pelo sistema "duplo-cego", garantido a imparcialidade na avaliação. Os avaliadores recebem um link para o artigo e respondem a um formulário-padrão de avaliação do artigo. 0 formulário de avaliação inclui questões relativas à: relevância do trabalho, estrutura do artigo, redação, fundamentação, método científico, apresentação e discussão dos resultados e conclusão, originalidade, adequação das figuras, tabela e quadros e formatação. Uma vez respondida todas questões do formulário e incluído comentários (caso desejado), o avaliador deve emitir uma entre as opções de recomendação apresentadas no Quadro 1.

\section{Quadro 1 -Opções de recomendação pelos Avaliadores}

\begin{tabular}{|l|l|}
\hline Recomendação & \multicolumn{1}{c|}{ Detalhamento } \\
\hline ACEITAR & aceitar o artigo como está \\
\hline REVISÕES REQUERIDAS & aceitar com revisões \\
\hline SUBMETER A NOVA RODADA & $\begin{array}{l}\text { sem decisão pois revisões significativas são } \\
\text { requeridas, demandando nova rodada de } \\
\text { avaliação, incorporando os comentários e } \\
\text { sugestões }\end{array}$ \\
\hline REJEITAR & rejeitar \\
\hline
\end{tabular}

Fonte: CILANO e BELTRANO (2018)

As recomendações (1) e (2) indicam um artigo aceito que deve seguir para a etapa de edição final. A recomendação (3) indica um artigo que deve ser reencaminhado para os autores com recomendações aguardando-se novo upload do artigo para nova rodada de revisão pelos avaliadores. A recomendação (4) indica rejeição sumária do artigo.

Um arquivo que incorpora sugestões dos avaliadores deve facilitar a identificação das mesmas. Portanto, recomenda-se que toda alteração no texto esteja identificada em FONTE AZUL ao se fazer novo upload de nova versão do artigo em resposta a um processo com recomendação do tipo 2 ou 3. Requer-se também a inclusão de uma carta aos editores explicando como as sugestões foram incorporadas no texto.

Havendo grande divergência na recomendação entre avaliadores solicita-se o parecer de um terceiro/quarto avaliador, seguindo o mesmo processo anterior.

\section{Conclusão}

Sendo o artigo aceito passa-se para a etapa de edição final do mesmo. Nesta etapa os editores têm o direito efetuar, nos originais aprovados para publicação, revisão e alterações de ordem normativa, ortográfica e gramatical. O processo total de publicação pode durar um período de até 3 meses.

A seção de conclusões não é obrigatória. Embora esta possa rever os pontos principais do artigo, a conclusão deve discorrer sobre a importância do trabalho ou sugerir aplicações e extensões. Indique de forma clara as vantagens, as limitações e 
as possíveis aplicações. As conclusões devem responder às questões da pesquisa, correspondentes aos objetivos e hipóteses; devem ser breves podendo apresentar recomendações e sugestões para trabalhos futuros.

\section{Citações e Referências}

A citação e a correspondente apresentação de referências concentram, normalmente, o maior número de inconformidades dos artigos. Nesta subseção serão apresentados exemplos de citações e referências com o objetivo de auxiliar os autores. Os padrões e exemplos apresentados na sequência foram baseados no sistema de chamada autor-data, conforme a NBR 6023-2002 e NBR 10520-2002. As Referências devem conter exclusivamente os autores e textos citados no trabalho e ser apresentadas ao final do texto, em ordem alfabética, obedecendo às normas atualizadas da ABNT. Materiais que não contenham as referências ou que as apresentem de forma incorreta não serão considerados para análise e publicação.

\subsection{Citações Indiretas}

As referências sem citações literais devem ser incorporadas ao texto, entre parênteses, indicando o sobrenome do autor em letras maiúsculas e o ano da publicação (SOBRENOME DO AUTOR, ano). A indicação de páginas é opcional, uma vez que a citação faz uma referência indireta à origem das ideias. Este parágrafo é um exemplo de uma citação indireta para um livro. Citações indiretas visam referenciar o texto no qual o autor baseou suas ideias (AUTOR, 1998).

\subsection{Citações Diretas}

Quando o autor citado integra a frase, só o ano e página serão colocados entre parênteses. Por exemplo, Autor (ano, p. xx-yy) é uma forma de citação direta. O sobrenome do autor, neste caso, terá apenas a primeira letra em maiúsculo, pois faz parte do texto. Este parágrafo é um exemplo de uma citação direta para um artigo e/ou matéria de um periódico. São exemplos de publicações periódicas: fascículo ou número de revista, número de jornal, artigos científicos de periódicos, editoriais, matérias jornalísticas, seções, reportagens, etc. Segundo relato publicado por Beltrano (2012, p. 12-16), as citações diretas são transcrições textuais de parte da obra do autor consultado

\subsection{Citações Diretas com mais de três linhas}

As citações diretas de mais de três linhas serão destacadas no texto em parágrafo especial, justificadas, com $3 \mathrm{~cm}$ de margem esquerda e espaçamento simples. Ao final, apresenta-se (SOBRENOME(S) DO(S) AUTOR(ES), ano, p. a-b). Como um exemplo de citação direta com mais de três linhas, ilustra-se a citação de uma monografia em meio eletrônico (livro e/ou folheto). A introdução apresentada por Fulano (2010) demonstra um alto domínio da língua:

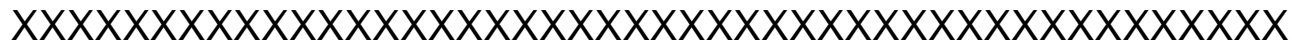

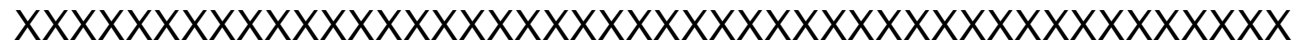

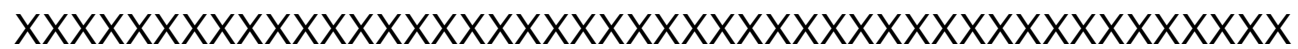

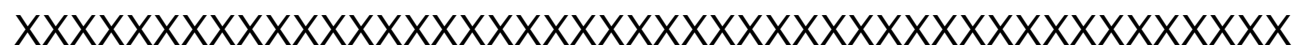
$X X X X X X X X X X X X X X X X X X X X X X X X X X X X X X X X X X X X X X X X X X X X X X$ $X X X X X X X X X X X X X X X X$ (FULANO, 2010, p. 10). 
Para as citações longas como a exemplificada acima, deve-se utilizar fonte em tamanho 12, espaçamento entre linhas simples, com recuo de 3 centímetros a partir da margem esquerda.

\subsection{Citações Diretas com menos de três linhas}

As transcrições literais curtas serão integradas ao parágrafo, colocadas entre aspas e seguidas pelo sobrenome do autor do referido texto (com letras maiúsculas), ano de publicação e página(s) do texto citado, entre parênteses (FULANO; BELTANO; CICLANO, ano, p. a-b). O texto em destaque deverá aparecer entre aspas, não sendo permitido a utilização de formatação em itálico, negrito ou sublinhado.

Este parágrafo é um exemplo de uma transcrição direta de até três linhas de um artigo de um periódico. $O$ estudo afirma que

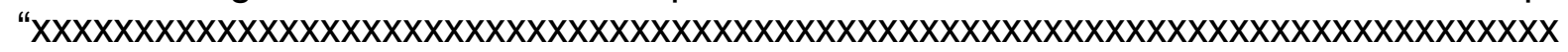


xxxxxxxxxx" (FULANO; BELTANO; CICLANO, 2017, p. 120).

\section{Notas de Rodapé}

Para facilitar o trabalho de formatação do artigo científico, recomenda-se evitar a criação de notas de rodapé, uma vez que o controle das mesmas não é algo muito fácil com o uso do programa MS Word. É usual encontrar problemas de notas que insistem em se posicionarem na página seguinte àquela desejada.

\section{Figuras e Tabelas}

Figuras e tabelas devem ser incluídas como parte do texto sempre que possível. Estes elementos não devem ser divididos em mais de uma página. Evite colocar as Figuras e Tabelas (ou Quadros) antes de sua primeira menção no texto. É desejável que as figuras tenham elementos coloridos e seus títulos devem ser posicionados depois das mesmas, em negrito. Quando houver mais de uma ilustração, a mesma deve ser numerada com algarismos arábicos, de modo crescente, precedido da palavra que a designa. Exemplo: Quadro 1 - Título, Fotografia 1 - Título, Gráfico 1 - Título. Sua localização deve ser na parte inferior da ilustração; ao utilizar-se de ilustrações extraídas de outras fontes, deve-se mencionar sua autoria, ano de publicação e página da publicação original e, fazer as Referências ao final do texto. A indicação da fonte consultada (após a ilustração, na parte inferior), é obrigatório mesmo que seja produção do próprio autor. A Figura 1 é um exemplo prático de inserção ao longo do texto.

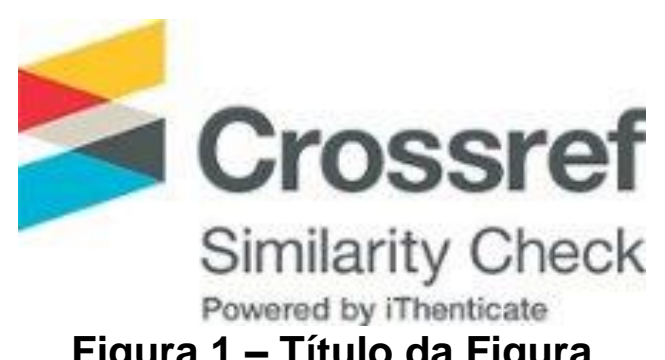

Figura 1 - Título da Figura

Fonte: O autor (2016) 
Para as Tabelas, o procedimento é diferente: seus títulos devem ser posicionados antes das mesmas, em negrito e centralizados. A fonte deve ser colocada imediatamente abaixo da tabela em letra maiúscula/minúscula para indicar a autoridade dos dados e/ou informações da tabela, precedida da palavra Fonte. Veja como exemplo a Tabela 1.

Tabela 1 - Dados da pesquisa de campo

\begin{tabular}{l|c|c|c|c}
\hline Entrevistado & \multicolumn{3}{|c|}{ Título Coluna [Unidade] } & Total \\
\hline- & $\mathrm{A}$ & $\mathrm{B}$ & $\mathrm{C}$ & - \\
\hline Entrevistado 1 & 10 & 2 & 3 & 15 \\
\hline Entrevistado 2 & 4 & 5 & 6 & 15 \\
\hline Entrevistado 3 & 7 & 8 & 9 & 24 \\
\hline Entrevistado 4 & 100 & 0 & 1 & 101 \\
\hline \multicolumn{5}{l}{ Fonte: O autor (2018). }
\end{tabular}

Observe que se deve declarar a fonte de todas figuras, tabelas e quadros, etc. Sendo a figura, tabela ou quadro (etc.) de autoria dos autores a fonte declarada é "O autor" ou "Os autores". Se a figura, tabela e quadro (etc.) é dos autores, mas já foram apresentados anteriormente em outro texto, deve-se fazer a citação ao texto original na declaração da fonte e incluir a referência na lista de referências do artigo. Se a figura, tabela ou quadro é de outro autor, para sua inclusão no artigo faz-se necessário ter a autorização de uso das mesmas. Não havendo a autorização de uso da figura, tabela ou quadro, orienta-se não os incluir no texto.

\section{Considerações Finais}

A intenção da REVES com este documento é facilitar o trabalho de todos os interessados. Além disso, abre-se um importante canal para o envio de críticas e sugestões, com objetivo de alimentar o processo contínuo de melhoria da qualidade do nosso trabalho. O contato deve ser estabelecido por meio do endereço eletrônico reves.journal@ufv.br.

Sendo o artigo aceito para a publicação com restrição, os autores devem acatar as recomendações dos pareceristas e providenciarem o envio da nova versão do artigo. Após a correção, a Comissão Editorial insere o nome do autor, enumera as páginas e demais dados pertinentes ao periódico, contendo informações sobre a edição da revista (volume, número, páginas etc.). Deve-se ressaltar que o artigo (originalidade, autoria, conteúdo abordado etc.) é de inteira responsabilidade do autor, assim como a apresentação do texto no padrão culto da língua. A Comissão Editorial se dá o direito de alterar a formatação e a linguagem do texto para ajustálas ao padrão da revista. A última etapa do processo editorial é a publicação da revista. A nova edição é disponibilizada com o Sumário, que elenca os títulos dos artigos, os respectivos autores e o link para o Resumo. Ao acessar o Resumo, o usuário pode baixar o artigo completo no formato PDF.

As referências devem ser apresentadas na sequência sob o título Referências, listadas em ordem alfabética seguindo a NBR 6023 (ASSOCIAÇÃO BRASILEIRA DE NORMAS TÉCNICAS, 2002b). Lembre-se que toda fonte bibliográfica citada no texto deve ser incluída nas referências. Adota-se na norma NBR 10520 (ASSOCIAÇÃO BRASILEIRA DE NORMAS TÉCNICAS, 2002) de citações em documentos. A exatidão das referências é de responsabilidade dos autores. As referências devem ser ordenadas alfabeticamente pelo sobrenome do autor, seguindo os padrões da norma NBR 6023 da ABNT, e não devem ser 
numeradas. As referências devem iniciar após o término da última seção do texto. Para as referências, deve-se utilizar texto com fonte ARIAL, tamanho 12, espaçamento SIMPLES, exatamente conforme aparece nas referências aleatórias incluídas a seguir. As referências, limitadas a 30, devem incluir apenas aquelas centrais e pertinentes à problemática abordada. Evitar a inclusão de número excessivo de referências numa mesma citação. Citações de documentos não publicados e não indexados na literatura científica (teses, relatórios e outros) devem ser evitadas. Caso não possam ser substituídas por outras, não farão parte da lista de referências. As referências deverão seguir as seguintes regras da ABNT (NBR 6023:2002), exemplificadas, conforme o tipo de material citados:

Se for Livro:

LÉVI-STRAUSS, Claude. As estruturas elementares do parentesco. Petrópolis: Vozes, 1982.

PRZEWORSKI, Adam; STOKES, Susan C.; MANIN, Bernard (Eds.). Democracy, accountability, and representation. New York: Cambridge University Press, 1999.

CALHOUN, Craig et al. (Eds.). Classical sociological theory. 2 ed. Malden, MA: Blackwell, 2007.

Se for Capítulo de livro:

WOLF, Eric. Etnicidade e nacionalidade. In: FELDMAN-BIANCO, Bela; RIBEIRO, Gustavo Lins (Orgs). Antropologia e poder: contribuições de Eric Wolf. Brasília/São Paulo: Ed.UnB/Imprensa Oficial, 2003.

Artigo em periódico:

BONELLI, Maria da Gloria; BARBALHO, Rennê Martins. O profissionalismo e a construção do gênero na advocacia paulista. Sociedade e Cultura, v. 11, n. 2, p. 275-284, 2008.

Se for Tese ou dissertação:

TAMASO, Izabela Maria. Em nome do patrimônio: representações e apropriações da cultura na cidade de Goiás. Brasília, 2007. Tese (Doutorado em Antropologia) Universidade de Brasília.

Se for Trabalhos apresentados em eventos:

BAQUERO, Marcelo. A democracia e capital social na América Latina: Brasil para além do debate acadêmico. In: CONGRESO LATINOAMERICANO DE CIENCIA POLÍTICA, 1, 2002, Salamanca. Política en América Latina:I Congreso Latinoamericano de Ciencia Política. Salamanca: Ediciones Universidad Salamanca, 2002. p. 837-858.

Se for Artigos em jornais:

BURITY, Joanildo A. Confronto de discursos. O Povo, Fortaleza, p. 6-7, 22 mar. 2008.

Texto disponível na internet:

MALINOWSKI, Bronislaw. The primitive economics of the Trobriand islanders. Economic Journal, vol. 31, p. 97-123, 1921. Disponível em: <https://socialsciences.mcmaster.ca/econ/ugcm/3\|l/3/malinowski/prim>. Acesso em: 08 jun. 2008. 
Ocorrências do mesmo autor:

Obras do mesmo autor publicadas no mesmo ano devem ser diferenciadas com o uso de letras logo após a data, sem espaço. Exemplo:

REIS, Fábio Wanderley. Mercado e utopia: teoria política e sociedade brasileira. São Paulo: Ed.USP, 2000a.

REIS, Fábio Wanderley. Política e racionalidade: problemas de teoria e método de uma sociologia 'crítica' da política. 2. ed. Belo Horizonte: Ed.UFMG, 2000b.

\section{Informações Relevantes}

(Recomenda-se o conhecimento prévio dos TUTORAIS, disponíveis no link https://periodicos.ufv.br/reves/Tutorials, bem como de INFORMAÇÕES ADICIONAIS - Equipe Editorial, Qualis, Taxas de Publicação, etc. - a respeito do periódico, disponíveis no link https://periodicos.ufv.br/reves/about).

\section{Agradecimentos}

Nesta seção final deve-se dedicar aos agradecimentos (opcional). Esta seção deve estar localizada entre o fim do corpo do texto e a lista de referências. Digite somente Agradecimentos em negrito, sem numeração, com alinhamento à esquerda e digite o texto na linha seguinte.

\section{REFERÊNCIAS}

BAQUERO, Marcelo. A democracia e capital social na América Latina: Brasil para além do debate acadêmico. In: CONGRESO LATINOAMERICANO DE CIENCIA POLÍTICA, 1, 2002, Salamanca. Política en América Latina:I Congreso Latinoamericano de Ciencia Política. Salamanca: Ediciones Universidad Salamanca, 2002. p. 837-858.

BONELLI, Maria da Gloria; BARBALHO, Rennê Martins. O profissionalismo e a construção do gênero na advocacia paulista. Sociedade e Cultura, v. 11, n. 2, p. 275-284, 2008.

BURITY, Joanildo A. Confronto de discursos. O Povo, Fortaleza, p. 6-7, 22 mar. 2008.

CALHOUN, Craig et al. (Eds.). Classical sociological theory. 2 ed. Malden, MA: Blackwell, 2007.

LÉVI-STRAUSS, Claude. As estruturas elementares do parentesco. Petrópolis: Vozes, 1982.

MALINOWSKI, Bronislaw. The primitive economics of the Trobriand islanders. Economic Journal, vol. 31, p. 97-123, 1921. Disponível em: $<$ https://socialsciences.mcmaster.ca/econ/ugcm/3ll3/malinowski/prim>. Acesso em: 08 jun. 2008.

PRZEWORSKI, Adam; STOKES, Susan C.; MANIN, Bernard (Eds.). Democracy, accountability, and representation. New York: Cambridge University Press, 1999.

REIS, Fábio Wanderley. Mercado e utopia: teoria política e sociedade brasileira. São Paulo: Ed.USP, 2000a.

REIS, Fábio Wanderley. Política e racionalidade: problemas de teoria e método de uma sociologia 'crítica' da política. 2. ed. Belo Horizonte: Ed.UFMG, 2000b.

TAMASO, Izabela Maria. Em nome do patrimônio: representações e apropriações da cultura na cidade de Goiás. Brasília, 2007. Tese (Doutorado em Antropologia) Universidade de Brasília. 
WOLF, Eric. Etnicidade e nacionalidade. In: FELDMAN-BIANCO, Bela; RIBEIRO, Gustavo Lins (Orgs). Antropologia e poder: contribuições de Eric Wolf. Brasília/São Paulo: Ed.UnB/Imprensa Oficial, 2003.

(Recomenda-se o conhecimento prévio dos TUTORAIS, disponíveis no link https://periodicos.ufv.br/reves/Tutorials, bem como de INFORMAÇÕES ADICIONAIS - Equipe Editorial, Qualis, Taxas de Publicação, etc. - a respeito do periódico, disponíveis no link https://periodicos.ufv.br/reves/about). 